\title{
The financial burden of imaging tests conducted in the emergency department of a teaching hospital: a brief report
}

\author{
Sahar Mirbaha' ${ }^{1}$, Mohammadmehdi Forouzanfar ${ }^{1}$, Amin Saberinia ${ }^{1}$, Amir Salimi², Hamid Kariman ${ }^{3}$, Mozhgan \\ Farmahini Farahani ${ }^{4^{*}}$
}

'Department of Emergency Medicine, Shohadaye Tajrish Hospital, Shahid Beheshti University of Medical Sciences, Tehran, Iran ${ }^{2}$ School of Medicine, Shahid Beheshti University of Medical Sciences, Tehran, Iran

${ }^{3}$ Department of Emergency Medicine, Imam Hosein Hospital, Shahid Beheshti University of Medical Sciences, Tehran, Iran

${ }^{4}$ Department of Emergency Medicine, Sina Hospital, Tehran University of Medical Sciences, Tehran, Iran

\begin{abstract}
Received: 11 July 2019
Accepted: 5 August 2019

Published online: 16 August 2019

*Corresponding author: Mozhgan Farmahini Farahani, Department of Emergency Medicine, Sina Hospital, Tehran University of Medical

Sciences, Tehran, Iran

Email: farahanimozhgan@yahoo. com

Competing interests: None.

Funding information: None.

Citation: Mirbaha S, Forouzanfar M, Saberinia A, Salimi A, Kariman $\mathrm{H}$, Farmahini Farahani M. The financial burden of imaging tests conducted in the emergency department of a teaching hospital: a brief report Journal of Emergency Practice and Trauma 2020; 6(1): 43-46. doi: 10.15171/jept.2019.14.
\end{abstract}

\begin{abstract}
Objective: Each imaging technique has a special application and usage, and should be used in the right situation. Physicians choose the type of imaging technique by considering the type of tissue and the benefits and disadvantages of the imaging method as well as its financial burden on the patient. The aim of this study was to estimate the cost of imaging tests performed in the emergency department (ED) of an educational hospital and determine their financial burden on both the patients and the healthcare system of the country.

Methods: This retrospective descriptive cross-sectional single-center study was conducted in Shohadaye Tajrish, an educational hospital, during one year. The study population consisted of all patients who had undergone some type of imaging (CT scan, ultrasound, radiography, magnetic resonance imaging [MRI]) in the ED. The information was collected using a predesigned checklist. The costs of a variety of imaging methods for patients referred to the ED were calculated using different types of accounting units and PACS system.

Results: The number of patients who visited the ED of the studied hospital during a one year period was 63507 . The total cost of performing different types of imaging methods in the studied center throughout the one-year target period was 44018695695 Rials $(\simeq \$$ US 423745 ) (59.27\% of which was spent on CT scan, $16.09 \%$ on ultrasound, $13.75 \%$ on plain radiography and $10.87 \%$ on $\mathrm{MRI}$ ).

Conclusion: According to the collected data, the total cost of radiology was 44018695695 Rials. The highest cost was related to CT scan modality, and the lowest belonged to MRI. Keywords: Costs, Cost analysis, Emergency service, Hospital, Radiology, Iran
\end{abstract}

\section{Introduction}

One of the most important units of each emergency department (ED) is the radiology unit, which plays an important role in the diagnostic process of patients. There are several types of radiologic imaging modalities and physicians choose the most appropriate one based on patients' condition. Of course, the costs of these methods are different. By measuring their prices and comparing them, we can determine their financial burden on patients and the healthcare system (1). It seems that the use of imaging modalities in ED is dramatically increasing $(2,3)$. In a 15 -year review, Li et al reported that computed tomography (CT) scan had the highest cost (4). Insufficient information about the imaging cost leads to overuse of radiological modalities. It could also lead to high depreciation of devices in health centers. On the other hand, one of the reasons for patients not going to the hospital and not pursuing healthcare is financial costs of imaging tests. With a simple review, we can estimate the amount of these costs and make a plan to reduce them (5). Therefore, this study was designed to determine the financial burden of the imaging tests conducted in the ED of an educational hospital on both the patients and the healthcare system.

\section{Methods}

This study is a retrospective cross-sectional, single center study conducted from April 2017 until March 2018 in 
Shohadaye Tajrish hospital, an educational hospital located in Tehran, Iran.

The study population consisted of all patients who underwent any type of imaging (CT scan, ultrasound, $\mathrm{X}$-ray, magnetic resonance imaging $[\mathrm{MRI}]$ ) in the ED. In this study, age and sex limitations were not considered, and no exclusion criteria were considered for the study.

Data collection was done using a checklist. This checklist consisted of data regarding the type of imaging method, insurance coverage and costs. The costs of various imaging methods (CT scan, MRI, ultrasound, and X-ray) performed for patients referred to the ED during the oneyear period were extracted from the hospital PACS system. Total cost of the conducted imagings and also each type of various modalities were calculated. Thereafter, the share of each type of imaging from the total income of the ED was calculated. All the costs are reported in Iranian Rial. In order to report costs in US dollars, each US dollar was calculated based on the average price of dollars reported by the Central Bank of Iran (1 US dollar $=103880$ Iranian Rials) during the period considered.

Statistical analysis was performed using SPSS software version 21. By comparing the cost of each method, the financial burden of each of the imaging methods was presented separately. To describe the continuous variables, mean, standard deviation, median, and intermediate quadrant were used. To describe the categorical variables, frequency and percentages were used.

\section{Results}

The number of patients who visited the ED of the studied hospital during the year the research was performed was 63507. The total cost of performing different types of imaging methods was 44018695695 Rials ( $\simeq$ \$US 423745); $59.27 \%$ was due to CT scan, $16.09 \%$ due to ultrasound, $13.75 \%$ due to plain X-ray, and $10.87 \%$ due to MRI. Accordingly, the highest income of the hospital through imaging belonged to CT scan modality and the lowest belonged to MRI. Details of the cost of imaging in various modalities are presented in Figure 1. The cost of imaging using different modalities was calculated by determining the patient's share of the total amount in Table 1 .

In Figure 2, the cost of each modality to the total income of the ED is shown and compared. Based on the findings, the income from CT scan was $22.04 \%$ of the total income of the ED. This rate was $5.98 \%$ for sonography, $5.11 \%$ for plain x-ray, and $4.04 \%$ for MRI.

The CT scan modality included 41 different types. In total, CT scans were done 28081 times (442 times per 1000 visits) for a total of 22472 patients (Figure 3). This indicates that it was repeated in 5609 cases. In 26710 times, one of the 8 most common types of CT scans was performed. Five people had no insurance and $99.98 \%$ of the cases were covered by insurance. The share of patients from the total cost was $5.97 \%$ (Table 1 ).

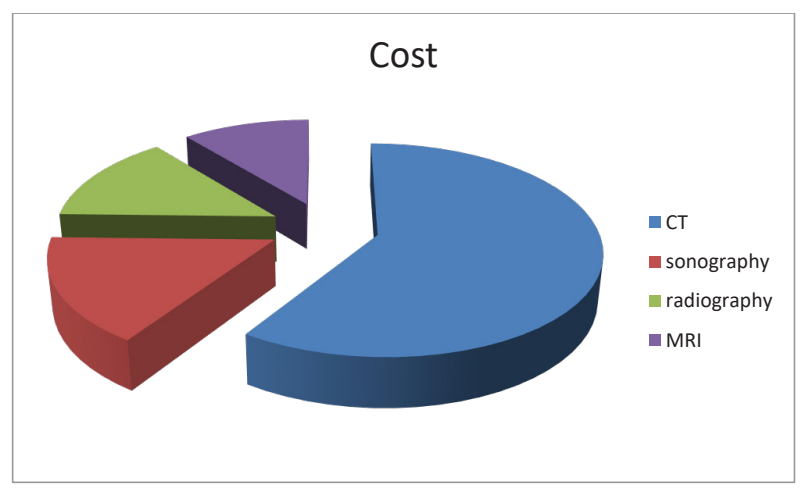

Figure 1. Contribution of each type of imaging to the total cost of imaging tests.

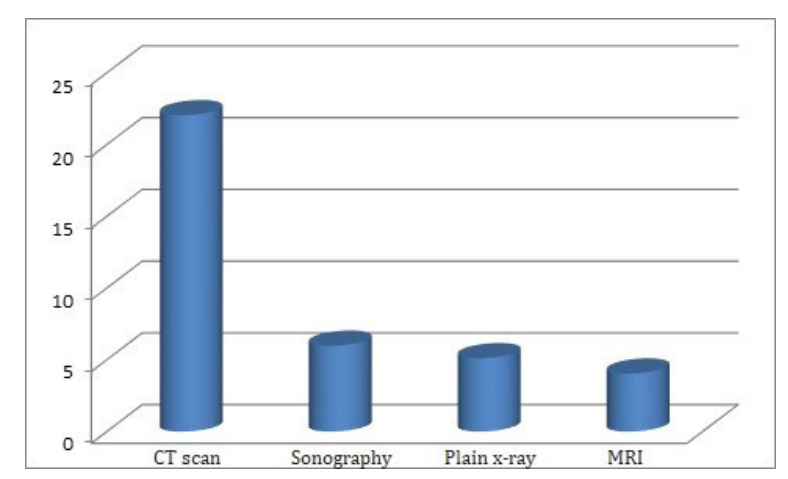

Figure 2. Percentage of the total income through each type of imaging to the total income of the emergency department.

Ultrasonography modality included 45 different types. Overall, ultrasonography was performed 14189 times (223 times per 1000 visits) for a total of 13886 patients, which means that it was repeated in 200 cases (Figure 4). In 11744 times, one of the 8 most common types of ultrasonography was carried out. Two people had no insurance and $99.98 \%$ of the cases were covered by insurance. The share of patients in the costs of this section was $10.03 \%$.

The plain radiography modality included 53 different types. A total of 35269 orders were placed for plain radiography (555 times per 1000 visits), which were performed on 33852 patients, this means that there were 1417 repetitions (Figure 5). In 28235 instances, 8 most common types of plain radiography were used. Three patients did not have insurance and $99.99 \%$ were covered by insurance. The share of patients in the costs of this section was $8.74 \%$.

The MRI modality included 27 different types. Overall, MRI was performed 4870 times (77 times per 1000 visits) for a total of 4810 patients, which means that there were 60 repetitions (Figure 6).. In 3957 times the MRI performed was one of the most common 5 MRI types. In this section, no one lacked insurance and $100 \%$ were covered by insurance. The share of patients in the costs of this section was $9.68 \%$. 
Table 1. The cost of imaging based on different modalities

\begin{tabular}{|c|c|c|c|c|}
\hline & \multicolumn{4}{|c|}{ Type of modality } \\
\hline & CT scan & Sonography & X-ray & MRI \\
\hline Total number & 28081 & 14189 & 35269 & 4870 \\
\hline Number per 1000 patient visits & 442.17 & 223.37 & 555.35 & 76.68 \\
\hline Total cost [Rials ( $\simeq$ US \$)] & $26093524807(2512)$ & $7082765856(6818)$ & $6053624692(5828)$ & $4788780340(4610)$ \\
\hline Patients' share (\%) & 5.97 & 10.03 & 8.74 & 9.68 \\
\hline
\end{tabular}

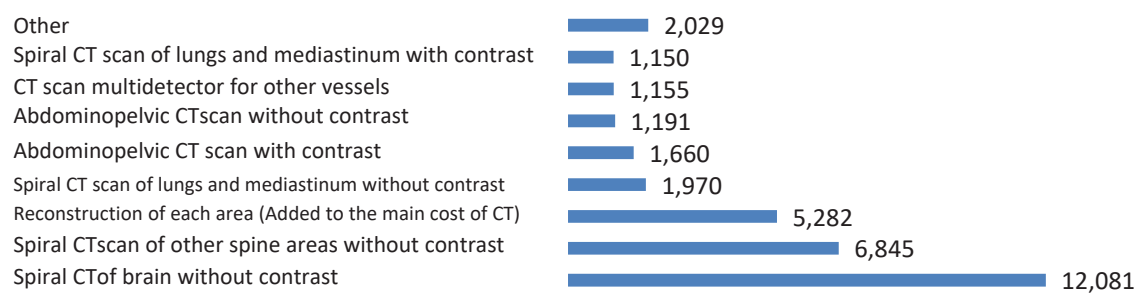

Figure 3. The frequency of CT scans performed in the emergency department

\begin{tabular}{|c|c|}
\hline Others & 2,442 \\
\hline & 707 \\
\hline Unilateral intravenous colour doppler ultrasound & $\begin{array}{r}752 \\
890\end{array}$ \\
\hline Appendix ultrasonography (pelvic- kidneys-appendix) & $\begin{array}{r}910 \\
950\end{array}$ \\
\hline Ultrasound of the liver and the gallbladder and biliary tract & 1,832 \\
\hline Ultrasound of the kidneys and urethra (Includes full bladder) & 3,187 \\
\hline
\end{tabular}

Figure 4. The frequency of ultrasonographies performed in the emergency department.

\begin{tabular}{|c|c|}
\hline Others & 8,034 \\
\hline & 888 \\
\hline Knee joint $x$-ray (Two ways -one film) & $\begin{array}{r}889 \\
-\quad 949\end{array}$ \\
\hline Foot x-ray (ap and lat- one film) & $\begin{array}{r}1,177 \\
1,338\end{array}$ \\
\hline Pelvic x-ray (every film) & $\begin{array}{l}2,091 \\
2,207\end{array}$ \\
\hline Chest x-ray (ap lat ) & 18,696 \\
\hline
\end{tabular}

Figure 5. The frequency of radiographies performed in the emergency department

\section{Discussion}

According to the present study, throughout the study period, the highest cost of radiological imaging modalities among the four main types performed in the ED belonged to CT scan, followed by ultrasonography and radiography; and MRI was considered to be the radiology modality with the lowest cost.

The study by Raja et al suggests that in recent years, the use of ultrasound and MRI has increased, and the use of CT scan and plain radiography has been reduced (6). However, according to data obtained from our study, in
Iran, CT scan is still used more than other modalities. In another study by $\mathrm{Li}$ et al, which was a comprehensive review of the past 15 years, CT scan had the highest incomes and costs in radiology throughout the year (4), which is similar to the result of our study.

The results of Ronkaien's paper reporting a shortterm study indicated that MRI was the most expensive radiology modality, but due to the greater use of CT scan compared to MRI, the highest cost and financial burden for the patients belonged to CT scan (7). This is also in line with the results of our research. In addition, in a study 


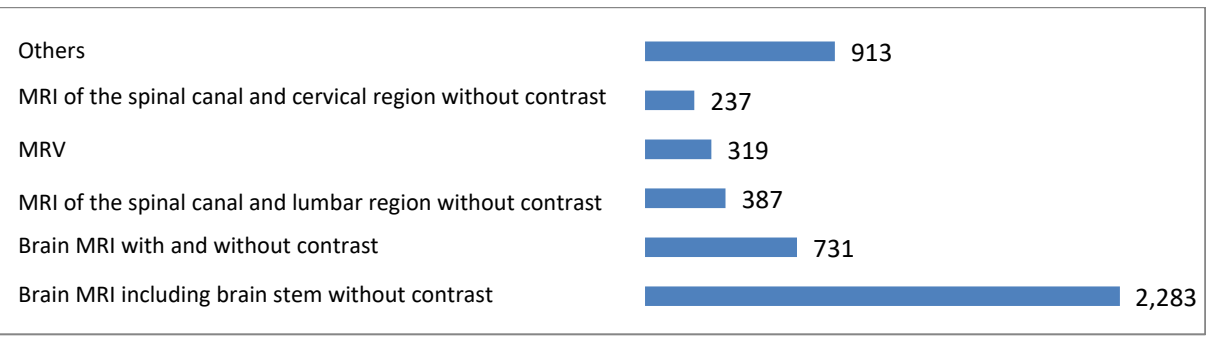

Figure 6. The frequency of MRIs performed in the emergency department.

by Rhea, a comparison was made between MRI and CT costs and just like our study, CT scan had the highest cost associated with radiology (8). In the study conducted by Hess et al in 2014, the use of CT had increased in the years 2000-2010 (9). Additionally, in the Oh HY study, in 2012, the use of the CT scan had increased by $255 \%$ (10). When comparing the statistics of the study by Quaday et al (11) with our study, the use of CT scan is higher in our center. Our study suggests that in addition to increasing the cost of treatment for patients, the use of radiological instruments can expose them to ionizing radiation, which has destructive effects, and will increase the wear and tear of the devices and reduce their lifespan, and all of these increase the cost of the healthcare in the country. Therefore, training of doctors on this subject is necessary.

\section{Conclusion}

According to the collected data, the total cost of radiology was 44018695695 Rials ( $\simeq$ \$US 423745 ) (59.27\% belonged to CT scan, $16.09 \%$ to ultrasonography, $13.75 \%$ to plain radiography, and $10.87 \%$ to $\mathrm{MRI})$. The highest income/ cost belonged to CT modality, and the lowest belonged to MRI. The rate of using these modalities in our center is much higher than other statistics reported.

Authors contribution

All the authors met the standards of authorship based on the recommendations of the International Committee of Medical Journal Editors.

\section{Ethical issues}

The study was ethically approved by the ethics committee of Shahid Beheshti University of Medical Sciences (ethics No. Ir.sbmu.msp.rec.1396.494). The ethical standards, including the confidentiality of the names of the patients under investigation, were observed at all stages of the implementation of this study.

\section{Acknowledgment}

Thanks to all the colleagues who worked on this project.

\section{References}

1. Al-Malki MA, Abulfaraj WH, Bhuiyan SI, Kinsara AA. A study on radiographic repeat rate data of several hospitals in Jeddah. Radiat Prot Dosimetry
2003; 103(4): 323-30. doi: 10.1093/oxfordjournals. rpd.a006149.

2. Mills AM, Raja AS, Marin JR. Optimizing diagnostic imaging in the emergency department. Acad Emerg Med 2015; 22(5): 625-31. doi: 10.1111/acem.12640.

3. Baratloo A, Talebian MT, Mirbaha S, Bagheri-Hariri S. Reducing Unnecessary CT Scanning in Head Trauma of Pediatric Patients: A Narrative Review. J Pediatr Rev 2019; 7(2): 99-104. doi: 10.32598/jpr.7.2.99.

4. Li Z, Zhang X, Müller H, Zhang S. Large-scale retrieval for medical image analytics: A comprehensive review. Med Image Anal 2018; 43: 66-84. doi: 10.1016/j. media.2017.09.007.

5. Lacy NL, Paulman A, Reuter MD, Lovejoy B. Why we don't come: patient perceptions on no-shows. Ann Fam Med 2004; 2(6): 541-5. doi: 10.1370/afm.123.

6. Raja AS, Ip IK, Sodickson AD, Walls RM, Seltzer SE, Kosowsky JM, et al. Radiology utilization in the emergency department: trends of the past 2 decades. AJR Am J Roentgenol 2014; 203(2): 355-60. doi: 10.2214/ajr.13.11892.

7. Ronkainen J. Costs in today's radiology: $\mathrm{aBC}$ analysis of typical situations in the transitional period. University of Oulu; 2007.

8. Rhea JT. Reducing hospital emergency department costs through aggressive use of diagnostic imaging. Emerg Radiol 1998; 5(3): 129-34. doi: 10.1007/ bf02749096.

9. Hess EP, Haas LR, Shah ND, Stroebel RJ, Denham CR, Swensen SJ. Trends in computed tomography utilization rates: a longitudinal practice-based study. J Patient Saf 2014; 10(1): 52-8. doi: 10.1097/ PTS.0b013e3182948b1a.

10. Oh HY, Kim EY, Kim JE, Kim YJ, Choi HY, Cho J, et al. Trends of CT use in the pediatric emergency department in a tertiary academic hospital of Korea during 2001-2010. Korean J Radiol 2012; 13(6): 7715. doi: 10.3348/kjr.2012.13.6.771.

11. Quaday KA, Salzman JG, Gordon BD. Magnetic resonance imaging and computed tomography utilization trends in an academic ED. Am J Emerg Med 2014; 32(6): 524-8. doi: 10.1016/j.ajem.2014.01.054. 